\title{
Heuristic Approaches with Energy Management for Node Placement in Wireless Sensor Networks
}

\author{
Sasikumar $P$ \\ Assistant Professor, VIT \\ Melasakkarapalayam, Vangal post \\ Karur, India - 639116
}

\author{
Shriram K Vasudevan \\ Assistant Professor, VIT \\ 68, Ram Nagar, 4th Cross, \\ Tanjore, Tamil Nadu, India. 613007
}

\author{
Ramesh M \\ Lecturer, \\ Sri Ramakrishna Engineering College \\ Vattamalaipalayam, N.G.G.O. \\ Colony Post, Coimbatore-641 022
}

\begin{abstract}
In Wireless Sensor Network, Device placement is a key factor for determining the coverage, connectivity, and cost along with lifetime. Managing the sensor nodes is not much easier while comparing mobile Ad $\neg$ Hoc Networks. But the same approach can be implemented to manage the WSN. Addressing the management of the whole network is omitted and a probabilistic scheme where only a subset of nodes is managed is provided for light $\neg$ weight and efficient management. Relay node placement in heterogeneous WSN are formulated using a generalized node placement optimization problem to minimize the network cost with lifetime constraint, and connectivity. Based on the constraints two scenarios are used. In the first scenario relay nodes are not energy constrained, and in the second scenario all nodes are energy limited. As an optimal solution a two-phase approach is proposed. The placement of the first phase relay nodes (FPRN), which are directly connected to Sensor Nodes $(\mathrm{SN})$, is modeled as a minimum set covering problem. To ensure the relaying of the traffic from the FPRN to the base station, three heuristic schemes are proposed to place the second phase relay nodes (SPRN). Some of the heuristic approaches available are Nearest $\neg$ to $\neg$ BS $\neg$ First algorithm (NTBF), Max $\neg$ Residual $\neg$ Capacity $\neg$ First algorithm (MRCF) and Best $\neg$ Effort $\neg$ Relaying algorithm (BER). Our contribution is centered on a distributed self organizing management algorithm at the application layer by organizing the management plane by extracting spatiotemporal components and by selecting manager nodes with several election mechanisms applied to wireless sensor nodes. Furthermore, a lower bound on the minimum number of SPRN required for connectivity is provided.
\end{abstract}

\section{Categories and Subject Descriptors}

D.3.2 [Programming Languages]: C++ : Language Contructs and Features - abstract data types, polymorphism, control structures.

\section{General Terms}

Algorithms, Management, Measurement, Documentation, Performance, Design, Economics, Reliability, Experimentation, Security, and Verification.

\section{Keywords}

Device placement, facility location problem, lifetime, minimum set covering, wireless sensor networks, Network management, probabilistic analysis.

\section{INTRODUCTION}

A Wireless Sensor Networks consists of three types of devices, Sensor Nodes (SNs), Relay Nodes (RNs), and a Base Station (BS), are used. In which the number and positions of sensing spots are deterministic and devices can be installed at deliberately chosen spots. The deployment of sensor nodes in the physical environment may take several forms. Nodes may be deployed at random (e.g., by dropping them from an aircraft) or installed at deliberately chosen spots. Deployment may be a onetime activity, where the installation and use of a sensor network are strictly separate activities. However, deployment may also be a continuous process, with more nodes being deployed at any time during the use of the network, for example, to replace failed nodes or improve coverage at certain interesting locations. The actual type of deployment affects important properties such as the expected node density, node locations, regular patterns in node locations, and the expected degree of network dynamics.

Depending on the actual needs of the application, the form factor of a single sensor node may vary from the size of a shoebox (e.g., a weather station) to a microscopically small particle (e.g., for military applications where sensor nodes should be almost invisible). Similarly, the cost of a single device may vary from hundreds of Euros (for networks of very few but powerful nodes) to a few cents (for large scale networks made up of very simple nodes). Varying size and cost constraints directly result in corresponding varying limits on the energy available (i.e., size, cost, and energy density of batteries or devices for energy scavenging), as well as on computing, storage and communication resources. Hence, the energy and other resources available on a sensor node may also vary greatly from system to system.

The remainder of this project is organized as follows. In chapter 2 , the sensor and system models are described. The general 
device placement problem with the objective of minimizing device cost under the constraints of coverage, connectivity and lifetime, is formulated. In chapter 3 , placement of relay nodes with and without energy constraints are preceded with proposed algorithms. Chapter 4 describes the key features that can be used to manage a WSN using probabilistic management, and the theoretical analysis to the localized heuristic algorithms are given in chapter 5. This project is concluded in chapter 6 with the performance evaluation and simulation results.

\section{Sensor and System Models}

\subsection{Sensor Model}

Sensor placement requires accurate yet computationally feasible sensor detection models. In this paper, first assume that the sensor field is made up of grid points. The granularity of the grid (distance between consecutive grid points) is determined by the accuracy with which the sensor placement is desired. Assume that the probability of detection of a target by a sensor varies exponentially with the distance between the target and the sensor

Figure1. Sensor Model

This model is illustrated in Fig. 1. A target at distance d from a

\section{Sensor Field}

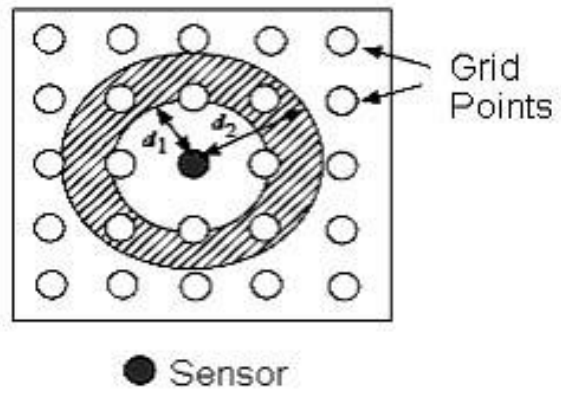

sensor is detected by that sensor with probability $\mathrm{e}^{-\alpha \mathrm{d}}$.

A number of sensors, e.g. IR cameras, require a target to lie in their line of sight. Obstacles cause occlusion and render such sensors ineffective for detection. We assume that some knowledge of the terrain is acquired prior to sensor placement, e.g. through satellite imagery. The obstacles are then modeled by altering the detection probabilities for appropriate pairs of grid points

\subsection{Network Model and Placement Problems}

Depending on the transmission range of RNs, we envision three types of scenarios:

Type I - RNs with adaptive transmission range, where the RNs can adjust their transmission power arbitrarily, so that the transmission from a source $\mathrm{RN}$ can reach any destination in the system;

Type II - RNs with fixed transmission range, where the RNs always transmit at a fixed power, thus the transmission from an RN can only reach other RNs that fall in its fixed transmission range; and Type III - RNs with limited adaptive transmission range, where the RN can adjust its transmission power within an upper bound.

Note that the energy supply of an $\mathrm{RN}$ can be limited or unlimited.

\subsection{Cost Model, Energy Model and System Lifetime}

The cost of a device depends on its functionalities and power supply. The more functionality a device and more complex and, thus, the more expensive it is. Also, there are various means of power supply (e.g., battery, solar panel, wall power) at different costs.

In this paper, we assume that the costs of individual nodes of the same type are the same. We adopt the communication power consumption model used in [6][7]. The energy consumption for receiving a packet of length $\mathrm{L}$ is

$$
j_{r x}=L \beta
$$

and the energy consumption for transmitting a packet of length $\mathrm{L}$ over the distance $\mathrm{d}$ is

$$
j_{t x}=L\left(\alpha_{1}+\alpha_{2} d^{m}\right)
$$

where $\alpha 1, \alpha 2$ and $\beta$ are hardware specific parameters, and $m$ is the path loss exponent.

\section{Placement of Relay Nodes}

First considering a basic version of the generalized problem, SNs are energy constrained and transmit data over a small distance. Individual SNs may have different transmission ranges depending on their initial energy and surrounding environment. RNs have large transmission distance and ample energy supply (wall powered, solar powered, or high capacity battery) so that their energy constraint is not a critical issue in contrast to the SNs. RNs can send data to the BS in one hop or multiple hops. In this scenario, as the connectivity and lifetime constraints on RNs are not factors, the optimization problem becomes: given a deployment of SNs, find a minimum number of RNs and their positions so that each $\mathrm{SN}$ can reach at least one $\mathrm{RN}$ in a single hop. Let $X=\left\{\mathrm{o}_{1}, \mathrm{o}_{2}, \ldots, \mathrm{o}_{\mathrm{N}}\right\}$ be the set of given SNs. If an RN is placed at a strategic position where multiple $\mathrm{SN}$ discs overlap, the corresponding SNs will all benefit from this RN. We make the following definitions,

Definition 1: Region. For a given set X of N SNs, let a subset s of $\mathrm{X}$ denote the intersection of the corresponding discs. Then $\mathrm{s}$ is called a region if this intersection is nonempty. For example, the subset $\mathrm{s}=\left\{\mathrm{o}_{1}, \mathrm{o}_{2}\right\}$ denotes the nonempty intersection of the discs of $\mathrm{O}_{1}$ and $\mathrm{O}_{2}$. An RN placed in the intersection corresponding to $\mathrm{s}_{\mathrm{i}}$ can serve at least as many $\mathrm{SNs}$ as an $\mathrm{RN}$ placed in the intersection corresponding to $s_{i}$ if $s_{j j} \subset s_{i}$, and vice versa. Note that each $\mathrm{SN}$ disc is also a region by itself.

Definition 2: Densest Region. A region si is said to be a densest region if there is no region, satisfying

$$
\mathrm{s}_{\mathrm{i}} \subset \mathrm{s}_{\mathrm{k}}
$$




\section{Probabilistic Management of WSN}

Probabilistic Management is one of the most popular management schemes for Monitoring and managing ad $\neg$ hoc networks is challenged by several constraints which are not encountered in the common fixed networks. Now in this paper as an extended work these are introduced in WSN [13]. The constraints encountered are

-
-
Management domain related
Management node reliability and willingness to
cooperate
Cost of monitoring

Probabilistic management is a selective scheme which consists in only managing the most interesting nodes of the network based on a statistical analysis. The notion of interesting is define with respect to relative good network presence and topology relationship. Spatio $\neg$ temporal component are considered to be a subset of the network nodes, such that any pair of nodes has a high adjacency probability. The term spatio $\neg$ temporal is inspired by the spatial dimension - nodes are required to be adjacent and the temporal one - nodes should have a high probability to be adjacent. Since this probability is estimated over a given time period, identify pairs of nodes that tend to directly interconnect. Such pairs can be aggregated in spatio-temporal components. This can be done by identifying all connected components in a graph built from the original network nodes, where an edge between two nodes is constructed if and only if a significant probability of adjacency for these nodes was estimated.

\subsection{Distributed Algorithmic Management Method}

The algorithmic method for probabilistic management is based on the spatio 7 temporal connectivity measure. A network node evaluates its spationtemporal connectivity with its neighborhood and communicates that information to the other network nodes in order to construct the spatio $\neg$ temporal connectivity matrix of the wireless sensor network. From this matrix, the node is capable to detect spatio $\neg$ temporal connected components of the network and to elect its network manager. We assume a minimal cooperation among nodes, where partial control is allowed. If necessary, the cooperation could be stimulated by considering an incentive approach such as [14]. Here in our work we are using a relay node for monitoring process such that a node that can efficiently communicate with all other nodes in a region.

\subsubsection{Spatio $\neg$ Temporal Connectivity Measure}

An WSN is seen as a set of $n$ mobile nodes $V=\left\{v_{1}, v_{2}, \ldots, v_{n}\right\}$ moving in a given surface during a time period $\mathrm{T}$. The time period $\mathrm{T}$ is split in $\mathrm{k}$ measurement interval $\left[\mathrm{t}_{1}, \mathrm{t}_{\mathrm{l}+1}\right]$ with

$$
T
$$

$t_{1}=l \times{ }_{k} \quad$ for an integer $1 \in[0, \mathrm{k}]$. Each node vi measures the percentage of time pij,, it was neighbor with a network node vi . On a time interval $\left[\mathrm{t}_{1}, \mathrm{t}_{\mathrm{l}+1}\right]$, the measurements are locally stored in a list.
$\mathrm{N}^{\mathrm{l}}$ (v) composed of tuples $\left(\mathrm{v}_{\mathrm{i}}, \mathrm{v}_{\mathrm{j}}, \mathrm{p}_{\mathrm{ij}}\right)$ and are subsequently exchanged and merged among network nodes. The suffix 1 represents the time factor and means the measure was performed during the time interval $\left[\mathrm{t}_{1}, \mathrm{t}_{1+1}\right]$. The exchange of local measurements leads conceptually to a network spatio $\neg$ temporal connectivity matrix $\mathrm{M}^{1}$ STC (or at least a partial view) obtained by concatenating the list of measurements performed by the network nodes. Rows/columns stand for a network node. The i $\neg$ th row of $\mathrm{M}_{\text {STC }}^{1}$ represents the list of measurements $\mathrm{N}^{1}\left(\mathrm{v}_{\mathrm{i}}\right)$ of a node $\mathrm{v}_{\mathrm{i}}$. If node $v_{i}$ was neighbor with node $v_{j}$ on $\left[t_{1}, t_{1+1}\right]$, an entry $\mathrm{M}_{\text {STC }}^{\mathrm{l}}[\mathrm{i}, \mathrm{j}]$ exists and contains the percentage of time pij that the pair $\left(\mathrm{v}_{\mathrm{i}}, \mathrm{v}_{\mathrm{j}}\right)$ was directly connected on that time interval. As the goal is to highlight network nodes presenting a high probability of adjacency (and also to limit the management data), only the spatio $\neg$ temporal connectivity values which are higher than a threshold value $\lambda$ are considered in the matrix.

\subsection{Algorithm for Extraction of Connected Components}

Data : Spatio-temporal connectivity matrix $\mathrm{M}_{\mathrm{STC}}^{1}$

Result : Connected component $C C_{v i}$ of node $\mathrm{v}_{\mathrm{i}}$

Initialization :

A) Initialize the set $C C_{v i}$ with the node $\quad\left\{\mathrm{v}_{\mathrm{i}}\right\}$ as the single element; //i.e. $C C_{v i}=\left\{v_{i}\right\}$

Repeat :

B) add to the set $C C_{v i}$ all the nodes connected to a node element of $C C_{v i}$;

C) delete all the doubles of $C C_{v i}$;

Until :

No change in calculation of connected $C C_{v i}$

\subsection{Manager election in a connected component}

A connected component, such as $\mathrm{CC}_{\mathrm{vi}}$, represents a set of network nodes with an high value of spatio $\neg$ temporal connectivity. It represents a sub-domain to be managed by one manager, or possibly by several managers if the connected component size is significant. An election mechanism is required to determine the manager nodes in each connected component. Assuming that each node in the network has a unique identifier (MAC address), the simplest way is to define an arbitrary election mechanism by electing the node with the minimal identifier. This mechanism is thus not efficient, as it does neither take into account the structural properties of the connected component nor the relative importance of nodes. We will define more refined election mechanisms based on centrality and Kmeans paradigm. These mechanisms will be applied to the data set formed by the spationtemporal connectivity measurements 
limited to the nodes in the connected component. This data set is a sub-matrix noted $\mathrm{S}_{\text {STC. }}^{1}$

\section{Localized Heuristic Placement Algorithm}

For an arbitrary placement of SNs, the solution space for placing RNs is infinitely large, and finding the optimal one is highly non $\neg$ trivial. A two - -phase topology design framework is proposed, wherein each phase decides the number and locations of RNs in a locally optimal fashion. The placement of first phase relay nodes (FPRNs) aims at ensuring the connectivity and lifetime of SNs with a minimum number of RNs. The placement of FPRNs was formulated as a minimum set cover problem, and an optimal recursive algorithm was proposed [15].

Since RNs are energy limited, in general FPRNs connecting to SNs are not able to transmit data to the BS by themselves. Thus, placement of second phase relay nodes (SPRNs) is needed to satisfy lifetime and connectivity requirements of FPRNs. In [15], two essential design principles, namely, the Far- $\neg$ Near and Max$\neg$ Min (FNMM) principles have been identified.

\subsection{Principles Used}

The Far $\neg-N e a r$ Principle refers to that the placement decisions in the second phase should first consider the RNs which are farthest from the BS and evolve step--by-רstep to the RNs that are closest to the BS. The rationale is that data are to be forwarded towards the BS. Hence RNs that are closer to the BS should relay the traffic for other farther nodes. This principle helps avoid energy wastage incurred due to unnecessary detouring of traffic relaying. The Max--Min Principle refers to maximally utilize the capacity of existing RNs, while introducing a minimum number of new RNs. Specifically, from far to near to the BS, each RN will distribute its workload to other existing neighboring RNs first. Only when the existing neighboring RNs of a given $\mathrm{RN}$ cannot support the traffic load from it, a new $\mathrm{RN}$ will be added.

The preliminary FNMM- based scheme in [15] is called the Nearest- $\neg$ To- $\neg$ BS- $\neg$ First algorithm (NTBF). We define the workload of a RN as the sum of its relayed traffic load, and its residual capacity as the difference between its capacity and its workload. Starting from the farthest RN, say far RN, if the workload of far RN does not exceed the total residual capacity of its adjacent neighbors, then its work-load is distributed to its adjacent neighbors, by first filling up the capacity of the node nearest to the BS, then to the node next nearest to the BS, and so on. In case two or more neighboring nodes having the same distance to BS, one is arbitrarily chosen. Otherwise, a new RN will be introduced as its next hop relay. The relay arrangement is recursively conducted until all RNs can find its next hop relay, or can reach the BS directly.

\subsection{Localized Heuristic Algorithms}

\section{Nearest-To-BS-First algorithm (NTBF)}

2. Max-Residual-Capacity-First algorithm (MRCF)

3. Best-Effort-Relaying algorithm (BER)

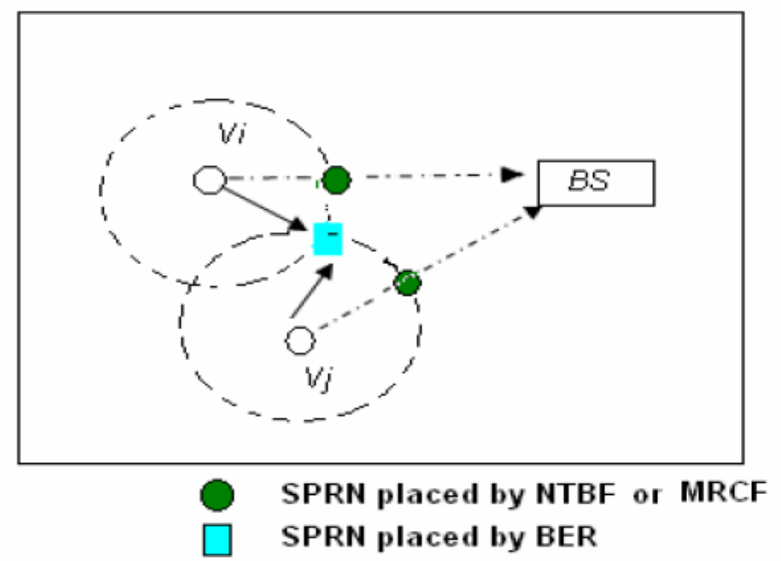

Figure 2. SPRN placement and traffic distribution by the three algorithms

\section{PERFORMANCE EVALUATION AND SIMULATION RESULTS}

From Fig.3, we observe the following.

1) Both algorithms require fewer SPRNs as the RN's capacity increases. This is a desirable property, as nodes with higher capacity should relay more traffic than those with lower capacity.

2) Using either algorithm, the ratio of the SPRNs obtained from experiments to the theoretical lower bound is $1.1 \neg 1.3$, which is quite acceptable.

3) The Best- $\neg$ Effort $\neg$-Relaying algorithm outperforms the Nearest-ᄀTo $\neg$-BS-First algorithm in terms of smaller number of SPRNs, higher utilization, and smaller C.O.V., though the improvement is moderate.

4) From Fig.3 (B) notice that with the increase of individual nodal capacity, the composite energy cost increases, while the resource utilization decreases. This suggests that an energy-economical system may prefer to use a larger number of devices with less energy rather than using a smaller number of devices with higher energy. This could be helpful for the network designer to choose the devices.

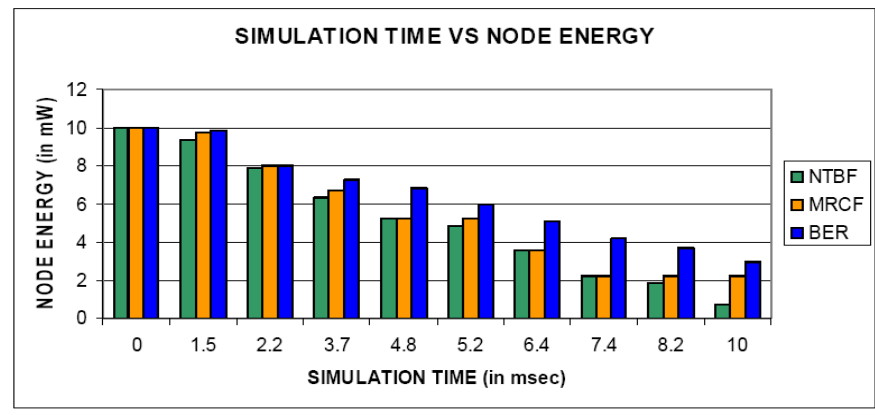

Figure 3(A) shows the energy level Vs Simulation Time for the three algorithms 


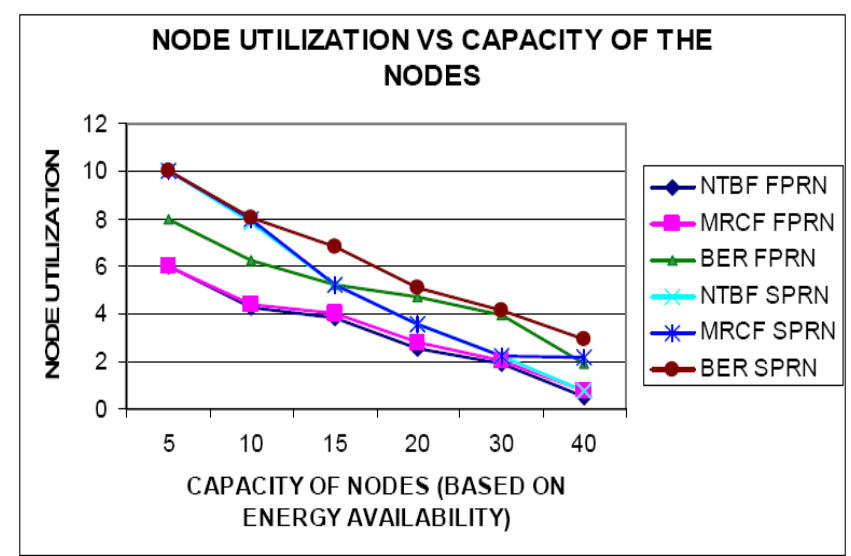

Figure 3(B). Shows the Node Utilization Vs Capacity of the Nodes

\section{CONCLUSION}

Here we have given the common problems of optimal WSN device placement, aiming at minimizing the network cost with constraints on lifetime and connectivity. A general design problem was formulated and discussed. The placement problem with non-ᄀenergy-ᄀconstrained relay nodes was modeled as a minimum set covering problem, taking into account the energy and transmission range constraints on RNs, a comprehensive two phase approach was presented. Based on the solution to problem one, an optimal solution was presented to place the first phase relay nodes. For the placement of second phase relay nodes, the Far ᄀ-Near and Max ᄀ-Min principles were proposed, and three heuristic schemes were used accordingly. For the management problems we have presented in this paper a new probabilistic scheme for configuring the management plane which is mostly used in an ad $\neg$ hoc network. The underlying key idea is the notion of spatio $\neg$ temporal connected nodes. A spatio $\neg$ temporal connected component is a subset of the wireless sensor network, such that nodes within such a component have a good probability of being directly connected. The term spatial derives from this neighborhood notion, while the term temporal is related to the temporal behavior of this neighborhood. In a store and forward oriented architecture, such nodes are also capable to inter $\neg$ communicate at a higher hop $\neg$-count. The management plane is limited to the largest spatio $\neg$ temporal connected components. Analysis and numerical results indicate that the proposed algorithms can provide optimal or near optimal solution in some scenarios. The relay node placement problem formulated in this paper is fundamental and critical in WSN design.

\section{ACKNOWLEDGEMENTS}

We acknowledge all the co authors who helped us in the project through out. Also we herewith thank all the sites which gave us great support in the form of materials.

\section{REFERENCES}

[1] H. Abelson et al., Mar. 2000, "Amorphous Computing," CACM, vol. 43, no. 5, pp. 74-82.

[2] Y. T. Hou, Y. Shi, H. D. Sherali, and S. F. Midkiff, Sep. 2005,"On energy provisioning and relay node placement for wireless sensor networks," IEEE Trans. Wireless Commun., vol. 4, no. 5, pp. 2579-2590.

[3] S. S. Pradhan, J.Kusuma, and K. Ramchandran, Mar. 2002, "Distributed compression in a dense sensor network," IEEE Signal Process. Mag., vol. 19,no. 2, pp. 51-60.

[4] A. Sayeed, D. Estrin, G. Pottie, and K. Ramchandran, Guest Editors, IEEE Journal on Selected Areas in CommunicationsSpecial Issue on Self organizing Distributed Collaborative Sensor Networks, to be published

[5] Wireless Medium Access Control (MAC) and Physical Layer (PHY) Specifications for Low $\neg$ Rate Wireless Personal Area Networks (LRWPANs) ,2003, IEEE Std. 802.15.4.

[6] J. Pan, Y. T. Hou, L. Cai, Y. Shi, and S. X. Shen, Sep. 2003, "Topology control for wireless sensor networks," in Proc. ACM MOBICOM, pp.286-299.

[7] W. Heinzelman, A. Chandrakasan, and H. Balakrishnan, "Energyefficient communication protocols for wireless microsensor networks," in Proc. 33rd Hawaii Int. Conf. Syst. Sciences, Jan. 2000, vol.8,pp.8020-8029.

[8] D. Bertsekas and R. Gallager, Data Networks,1992, 2nd ed. Upper Saddle River, NJ:Prentice Hall.

[9] C. V. Ramamoorthy and B. W. Wah, Mar.1983, "The isomorphism of simple file allocation," IEEE Trans.Comput., vol. 32, no. 3, pp. 221-231.

[10] G. C. Armour and E. S. Buffa. Jan 963, "A heuristic algorithm and simulation approach to relative location in facilities," Manag. Sci., vol. 9, no. 1, pp. 294-309,.

[11] A. S. Manne, Nov. 1964, "Plant location under economies of scale decentralization and computation," Manag. Sci., vol. 11, pp. 213-235.

[12] M. Patel, R. Chandrasekaran, and S. Venkatesan, Apr. 2005, "Energy efficient sensor, relay and base station placements for coverage, connectivity and routing," in Proc. IEEE IPCCC, pp. 581-586.

[13] Remi Badonnel, Radu State, Olivier Festor, , JUNE 2007, A Probabilistic Approach for Managing Mobile Ad-Hoc Networks, IEEE TRANSACTIONS ON NETWORK AND SERVICE MANAGEMENT, VOL. 4,NO. 1.

[14] L. Buttyan and J. P. Hubaux, Oct. 2003, "Stimulating Cooperation in Self $\neg$ Organizing Mobile Ad Hoc Networks," ACM/Kluwer Mobile Networks and Applications, vol. 8, no. 5.

[15] Q. Wang, K. Xu, G. Takahara, and H. Hassanein, Nov.Dec. 2005, "Locally optimal relay node placement in heterogeneous wireless sensor networks," in Proc.IEEE GLOBECOM, vol. 6, pp. 3549-3553. 